\title{
Herbal medicines
}

\section{Pirmohamed}

\section{Always ask your patient about herbal medicines}

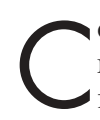

omplementary and alternative medicine (CAM) is a generic term for a range of therapies and practices including herbal medicine and acupuncture. Herbal medicines are the most widely used form of CAM. ${ }^{1}$ The general public has a more positive view of herbal medicines compared with conventional medicines, largely because they are perceived as being "natural" and "safe". However, recent events have shown that herbal medicines can have severe adverse health consequences. For example, aristolochia and kava kava have recently been withdrawn from the UK market because of their association with nephrotoxicity and hepatotoxicity, respectively. ${ }^{2}$ Similarly, regulatory action had to be instituted against St John's Wort because of its potential to interact with other medicines which led to serious adverse events such as graft rejection (interaction with immunosuppressants) and failure to suppress HIV (interaction with protease inhibitors). ${ }^{3}$

Ginkgo biloba extract is a widely used herbal preparation derived from the broad leaves of the maidenhair tree. It is advertised as being effective in a wide range of conditions including dementia, peripheral vascular disease, erectile dysfunction, premenstrual syndrome, asthma, senile macular degeneration, tinnitus, and vertigo. However, evidence of efficacy in these conditions is either lacking or at best inconsistent, and based on poorly designed trials with subjective end points. ${ }^{4}$ A variety of adverse effects have been reported with Ginkgo biloba extract including headaches, diarrhoea, and vomiting. The most serious adverse effect reported is haemorrhage, which has been attributed to the ability of ginkgolides, a component of the extract, to antagonise platelet activating factor." In this issue of the journal, Fong and Kinnear report the case of a patient on Ginkgo biloba extract who developed an acute retrobulbar haemorrhage after a peribulbar injection. ${ }^{6}$ This report, taken together with other reports of cerebral and extracerebral haemorrhage (see their table $1^{6}$ ), and the evidence of biological plausibility, ${ }^{5}$ provide a strong indication that the extract may predispose certain patients to bleeding episodes. Furthermore, patients on other drugs affecting either platelet function (non-steroidal anti-inflammatory drugs and clopidogrel) and/or coagulation (warfarin and heparin) are likely to be at higher risk of haemorrhage and thus the use of Ginkgo biloba extract should be contraindicated in these patients. The possibility of interactions with herbal medicines was starkly highlighted by the case of St John's Wort, ${ }^{3}$ but despite this there are indications that pharmaceutical companies do not routinely incorporate herbal medicines in their interaction studies of new chemical entities. ${ }^{\text {? }}$

Chemical analysis of different batches of Ginkgo biloba extract produced by the same manufacturer and that produced by different manufacturers is likely to show marked variation in the content and concentrations of the pharmacologically active constituents. By extrapolation, this will lead to variability in biological effects, and thus the risk of haemorrhage. Variability in composition is a problem seen with most herbal preparations since they are not manufactured in accordance with the principles of good manufacturing practice. ${ }^{1}$ Standardisation, however, may be difficult to achieve for some preparations because the active constituents may not be known. Contamination of herbal medicines is also recognised to be a problem; this may involve contamination with other toxic herbs, for example aristolochia, or with conventional drugs, for example, corticosteroids. ${ }^{2}$ Regulation of herbal medicines has to date been far from ideal, and does not give adequate protection against poor quality products. In order to improve the situation, the European Union has published a draft directive that aims to establish a harmonised legislative framework for authorising the marketing of herbal medicines. ${ }^{8}$ One important requirement of the directive, which will be in force by the end of 2004, is that the manufacturer of the herbal product will have to provide evidence demonstrating that the product has been manufactured according to the principles of good manufacturing practice. This will undoubtedly lead to improvements in the quality of products available to the public and, importantly through standardisation, in the assessment of efficacy of these compounds through high quality randomised controlled trials.

While we await these developments, what are the implications for clinical practice? First, it is important to realise that herbal preparations contain biologically active compounds, which may cause adverse effects and/or interact with concomitantly administered conventional drugs. ${ }^{4}$ Second, the use of CAM is widespread. About $22 \%$ of questionnaire respondents in the UK had purchased over-the-counter herbal or homoeopathic remedies in the previous year," while in the US, $43 \%$ had used CAM at some time. ${ }^{10}$ Approximately, one third of patients undergoing surgery admit to using herbal medicines. ${ }^{11} \mathrm{~Pa}-$ tients do not inform their doctors that they are taking such therapies, and importantly doctors do not routinely ask about CAM. It is therefore essential to document whether a patient is taking herbal medicines during any clinical evaluation; this may help in making a diagnosis when the patient develops new clinical features, and may help in avoiding serious interactions with conventional drugs.

\section{Postgrad Med J 2003;79:489}

Correspondence to: Professor Munir

Pirmohamed, Department of Pharmacology and Therapeutics, University of Liverpool, Ashton Street, Liverpool, L69 3GE, UK; munirp@liv.ac.uk

\section{REFERENCES}

1 Barnes J. Quality, efficacy and safety of complementary medicines: fashions, facts and the future. Part I: regulation and quality. $\mathrm{Br} J$ Clin Pharmacol 2003:55:226-33.

2 Barnes J. Quality, efficacy and safety of complementary medicines: fashions, facts and the future. Part II: efficacy and safety. Br J Clin Pharmacol 2003;55:331-40.

3 loannides C. Pharmacokinetic interactions between herbal remedies and medicinal drugs. Xenobiotica 2002;32:451-78.

4 De Smet PA. Herbal remedies. N Engl J Med 2002;347:2046-56.

5 Koltai M, Hosford D, Guinot P, et al. Platelet activating factor (PAF). A review of its effects, antagonists and possible future clinical implications (part I). Drugs 1991;42:9-29.

6 Fong KCS, Kinnear PE. Ginkgo biloba and retrobulbar haemorrhage following peribulbar retrobulbar haemorrhage following peribulbar local anaesthetic injection for catract
Postgrad Med J 2003;79:531-2.

7 Thompson Coon J, Pittler M, Ernst E. Herb-drug interactions: survey of leading pharmaceutical/herbal companies. Arch Intern Med 2003;163:1371.

8 Commission of the European Communities. Proposal for amending the Communities. Proposal for amending the
directive $2001 / 83 / E C$ as regards traditional herbal medicinal products. Brussels: European Commission, 2002.

9 Thomas KJ, Nicholl JP, Coleman P. Use and expenditure on complementary medicine in England: a population based survey. Complement Ther Med 200 1:9:2-1 1 .

10 Eisenberg DM, Davis RB, Ettner SL, et al. Trends in alternative medicine use in the United States, 1990-1997: results of a follow-up national survey. JAMA 1998;280:1569-75

11 Kaye AD, Clarke RC, Sabar R, et al. Herbal medicines: current trends in anesthesiology practice-a hospital survey. J Clin Anesth practice-a hospit 